\title{
Functional Impairment of Reading in Patients with Dry Eye
}

Priya M. Mathews, MD, $\mathrm{MPH}^{1}$; Pradeep Y. Ramulu, $\mathrm{MD}, \mathrm{PhD}^{1}$; Bonnielin S. Swenor, $\mathrm{PhD}^{1}$; Canan A. Utine, $\mathrm{MD}^{1,2}$; Gary S. Rubin, $\mathrm{PhD}^{3,4}$; Esen K. Akpek, MD ${ }^{1}$

${ }^{1}$ The Wilmer Eye Institute, Johns Hopkins University, Baltimore, Maryland, USA.

${ }^{2}$ Dokuz Eylul University, Department of Ophthalmology, Izmir, Turkey.

${ }^{3}$ Institute of Ophthalmology, University College of London, London, UK.

${ }^{4}$ Biomedical Research Centre for Ophthalmology, London, UK.

Running Title: Dry eye and reading impairment

Key words: quality of life, reading, functional impairment, dry eye.

Word Count: 3,777

\section{Address for Correspondence:}

Esen Karamursel Akpek, MD

Director, Ocular Surface Diseases and Dry Eye Clinic

The Wilmer Eye Institute

600 North Wolfe Street, Maumenee Building \#317

Baltimore, Maryland, 21287-9238.

Phone \#: (410) 9555214 Fax \#: (410) $6146480 \quad$ e-mail: esakpek@jhmi.edu 


\section{SUBTITLE}

2 Dry eye is associated with slower out-loud and silent reading. The decrement in reading speed

3 directly correlates with the severity of dry eye disease, as measured by the Ocular Surface

4 Disease Index and corneal staining score. 
ABSTRACT

Background/Aims: To evaluate the impact of dry eye on reading performance.

7 Methods: Out-loud and silent reading in patients with clinically significant dry eye $(\mathrm{n}=41)$ and

8 controls $(n=50)$ was evaluated using standardized texts. Dry eye measures included tear film

9 break-up time, Schirmer's test, and corneal epithelial staining. Symptoms were assessed by the

10 Ocular Surface Disease Index.

11 Results: The dry eye group had a greater proportion of women as compared to the control group

12 but did not differ in age, race, education level, or visual acuity ( $p \geq 0.05$ for all). Out-loud

13 reading speed averaged 148 words per minute (wpm) in dry eye subjects and $163 \mathrm{wpm}$ in

14 controls $(\mathrm{p}=0.006)$. Prolonged silent reading speed averaged $199 \mathrm{wpm}$ in dry eye subjects versus

$15226 \mathrm{wpm}$ in controls ( $\mathrm{p}=0.03$ ). In multivariable regression models, out-loud and sustained silent

16 reading speeds were $10 \mathrm{wpm}(95 \% \mathrm{CI}=-20$ to $-1 \mathrm{wpm}, \mathrm{p}=0.039)$ and $14 \%(95 \% \mathrm{CI}=-25 \%$ to -

$172 \%, \mathrm{p}=0.032$ ) slower, respectively, in dry eye subjects as compared with controls. Greater

18 corneal staining was associated with slower out-loud (-2 wpm/1 unit increase in staining score,

$1995 \% \mathrm{CI}=-3$ to $-0.3 \mathrm{wpm})$ and silent $(-2 \%, 95 \% \mathrm{CI}=-4$ to $-0.6 \mathrm{wpm})$ reading speeds $(\mathrm{p}<0.02$ for

20 both). Significant interactions were found between OSDI score and word-specific features

21 (longer and less commonly used words) on out-loud reading speed ( $\mathrm{p}<0.05$ for both).

22 Conclusions: Dry eye is associated with slower out-loud and silent reading speeds, providing

23 direct evidence regarding the functional impact of dry eye. Reading speed represents a

24 measurable clinical finding that correlates directly with dry eye severity. 

37 different previously validated texts.

\section{4}

\section{INTRODUCTION}

\section{MATERIALS AND METHODS} and January 2012.

\section{Study Subjects}

Dry eye is a common condition affecting approximately one in three individuals over the age of 50.[1-4] Although ocular discomfort may be the most bothersome symptom, visual complaints are also common. Dry eye has a substantial yet often under-appreciated impact on vision-related quality of life.[5-6] Prior research has shown that dry eye patients report difficulty in various vision-related tasks such as driving, reading, computer work, watching television, and performing work-related activities.[7-11] Arguably the most common visual complaint reported is difficulty with reading, which may affect employment or decrease work productivity.

In a population-based sample of elderly, we previously noted that dry eye symptoms were associated with greater perceived difficulty with reading and also the avoidance of specific reading tasks.[12] Here, we designed a clinical study to quantify reading performance through measuring actual reading speed on both a full-passage and individual word level by using several

The study protocol was approved by the Johns Hopkins University Institutional Review Board in accordance with the Declaration of Helsinki and was Health Insurance Portability and Accountability Act compliant. Study subjects completed the study procedures between July 2009

Eligible subjects had to be 50 years or older, literate by self-report, and able to communicate in English. Dry eye subjects were recruited from the Ocular Surface Diseases Clinic at Wilmer Eye Institute and had: (1) clinically significant dry eye defined as Schirmer's 
48 test result without anesthesia $\leq 7 \mathrm{~mm}$ at 5 minutes and/or bulbar conjunctival staining with

49 lissamine green $\geq 1$ on the Oxford scale in either eye [13], and (2) an Ocular Surface Disease

50 Index (OSDI) score of 13 or higher. All patients were on topical treatment at the time of

51 enrollment (including artificial tears and/or anti-inflammatories), which was not held prior to

52 testing.

53 Control subjects were gathered from individuals followed for suspicion of glaucoma at

54 the Glaucoma Clinic of the Wilmer Eye Institute who had (1) never been diagnosed with dry eye,

55 and (2) had an OSDI score of 12 or less. All controls had normal visual fields in both eyes over

56 the central 24 degrees using a size III stimulus and assessed by the Swedish interactive threshold

57 algorithm standard testing program (HFA2, Carl Zeiss Meditec Inc., Dublin CA). Thirty-one

$58(62 \%)$ of the control subjects were on intraocular pressure-lowering drops at the time of

59 enrollment, which was not held prior to testing.

60 Tests Performed

61

All subjects were examined in a uniform manner using the tests performed on a single

62 day in the following order:

63 Evaluation of Vision and Covariates

64 Sociodemographic variables were gathered using standardized forms. Visual acuity was

65 measured binocularly with patients' habitual distance correction using the Early Treatment

66 Diabetic Retinopathy Study vision chart, and summarized as the negative logarithm of the

67 minimum angle of resolution(logMAR).[14,15] All subjects had at least 20/40 or better vision in

68 both eyes.

Contrast sensitivity was measured using the Pelli-Robson chart under binocular

70 conditions and converted to a log scale.[16] The presence of depressive symptoms was assessed 
71 using part D of the General Health Questionnaire.[17] Cognitive ability was evaluated using the

72 Mini Mental State Exam (MMSE).[18] After reading tests were administered, pupils were

73 pharmacologically dilated and lens changes were graded as present or absent as described

74 previously.[19]

75 Evaluation of Reading

76 Subjects wore their habitual reading correction for the following assessments: (a) out-

77 loud reading speed using the Minnesota low vision reading test (MNRead)[20], (b) out-loud

78 reading speed using a 77-word international reading speed test (IReST)[21], and (c) sustained

79 silent reading speed using a 7,300-word validated passage read silently for 30 minutes or until

80 the passage is finished. Greater detail regarding the administration of these three reading tests is

81 provided elsewhere.[22]

82 Reading speed was calculated in words per minute (wpm). Maximum reading speed was

83 calculated from MNRead times using nonlinear mixed effects models.[23] IReST passage

84 reading speed was calculated after adjusting for reading errors. Sustained silent reading speed

85 was calculated from the total words read and time required for reading. Details regarding these

86 parameters are provided elsewhere.[22,23]

87 Evaluation of Word-specific Reading Data

Audiorecordings of the IReST passage were imported into Wave Editor Version 1.5.5

89 (Audiofile Engineering, Minneapolis, MN) and analyzed by a masked evaluator. The start and

90 end of each individual word was determined using the software spectrogram, and then imported

91 into a separate database to calculate the exact duration to say each word out-loud and the

92 following interval duration (before the start of the next word). Each word was analyzed as a

93 word plus post-word interval unit to capture any potential interactional effect of the word-level 
94 feature (i.e. word length, word frequency, and location of word in text). A detailed description

95 of the derivation of these outcomes is described in detail elsewhere.[24]

96

97

98

99

100

101

102

103

104

105

106

107

108

109

110

111

112

113

114

115

116

\section{Dry Eye Evaluations}

The Ocular Surface Disease Index (OSDI) questionnaire was administered to all subjects by a masked examiner.[10] Total scores were categorized for severity (normal=0 -12 , mild=1322, moderate $=23-32$, severe $=33-100) .[25,26]$ A similar formula was used to compute two OSDI subscores: 1) vision-related subscore corresponding to questions 4-9 assessing the impact of dry eye on visual functioning, and 2) ocular discomfort-related subscore corresponding to questions 1-3 and 10-12 evaluating symptoms relating to irritation or discomfort.[27] Subscale scores ranged from 0 to 50 .

Dry eye signs was assessed by one of three masked examiners (EKA, PYR, or CAU) and in the order listed here. Tear film break-up time (TBUT) was measured with 5 microliters of anesthetic-free preservative-free $2 \%$ sodium fluorescein using the cobalt blue light of a slit lamp and a Wratten 12 yellow filter 1 minute after instilling the eye drop. Three TBUT measurements were obtained (maximum value of 10 seconds) and averaged for each eye.

Corneal staining was evaluated using the National Eye Institute grading system. Within 2 to 3 minutes after TBUT testing, the extent of punctate epithelial erosions was graded using Wratten 12 filter paper.[28] Total corneal staining grade for each eye ranged from 0-15. Lastly, Schirmer's test was performed without anesthesia in each eye at least 10 minutes after corneal staining assessment, read at 5 minutes, and averaged.[29]

\section{Statistical Methods}

Group differences in demographic, health, and visual features were assessed using the Student's t-test for normally-distributed continuous variables, Wilcoxon rank sum testing for 
117 non-normally distributed continuous variables, and chi-squared testing for categorical variables.

118 The worse eye values for the TBUT, corneal staining, and Schirmer's test were used for the data

119 analysis. Variables associated with MNRead and IReST reading speeds were evaluated using

120 age-adjusted and multivariable linear regression models adjusting for age, sex, race, education,

121 employment status, cognitive ability, and the presence of depressive symptoms. Sustained silent

122 reading speeds were log-transformed and analyzed in age-adjusted and multivariable linear

123 regression models in order to obtain normally-distributed residuals. The percent change in log

124 sustained silent reading speeds associated with model elements was calculated as $\left(10^{(\beta)-} 1\right)^{*} 100$.

125 Predictors of the word/post-word interval unit were evaluated using multivariate linear

126 regression models. Covariates were included in multivariate models if they demonstrated a

127 significant impact on word time in age-adjusted models or had been previously shown to impact

128 reading speed.[30] Word features (i.e. word size, word frequency, location in text) were also

129 included in multivariable models. Lastly, GEE multivariate models were used to assess

130 interactions between dry eye severity and word features on word/post-word interval time. This

131 interaction analysis was included to evaluate whether dry eye patients had particular difficulty

132 with certain text features, similar to glaucoma patients.[24] All data were analyzed using STATA

133 statistical software (STATA Release 12.1; STATA Corp., College Station, TX).

$135 \underline{\text { RESULTS }}$

$136 \quad$ Forty-one dry eye patients and 50 controls completed study procedures and were included

137 for analysis. One patient was excluded based on a greater than 2-fold difference between their

138 silent and out-loud reading speeds. 
Participant characteristics are summarized in Table 1. There was no difference between

140 the two groups with regards to sociodemographic characteristics, cognitive ability, depressive

141 symptoms, presence of cataracts/posterior capsular opacity, visual acuity, or contrast sensitivity.

142 Women formed a greater proportion of the dry eye subject group as compared to the control

143 group (90\% vs. 58\%, p=0.001). Subjects with dry eye had significantly greater total (39.5 vs. 4.7,

$144 \mathrm{p}<0.001)$, ocular discomfort-related $(22.2$ vs. $2.8, \mathrm{p}<0.001)$ and vision-related (17.3 vs. 1.8,

$145 \mathrm{p}<0.001)$ OSDI scores than controls, in addition to shorter TBUTs (1.9 vs. 3.3 seconds, $\mathrm{p}=0.01)$

146 and greater corneal fluorescein staining (7.4 vs. 5.2, $\mathrm{p}=0.007)$. Schirmer's test without anesthesia

147 did not differ between the two groups $(\mathrm{p}=0.41)$.

148

149

controls for the IReST passage (148 vs. 163 wpm, p=0.006) and sustained silent reading (199 vs.

150

226 wpm, $\mathrm{p}=0.03$ ) but did not demonstrate slower maximum reading speeds in the MNRead test

151 (180 vs. 186 wpm, $\mathrm{p}=0.22)$ (Table 2). No significant differences were noted in other MNRead

152 parameters including reading acuity and critical print size ( $\mathrm{p}>0.05$ for both)(Table 2).

In multivariable models, dry eye was associated with significantly reduced IReST

154 passage reading speed $(-10 \mathrm{wpm}, 95 \% \mathrm{CI}=-20$ to $-1 \mathrm{wpm}, \mathrm{p}=0.04)$ and sustained silent reading

155 speed ( $14 \%$ slower, $95 \% \mathrm{CI}=-25$ to $-1 \%, \mathrm{p}=0.03)$, but not with a slower maximum MNRead

156 speed (Table 3). In separate multivariable models, reduction in the MNRead, IReST, and

157 sustained silent reading speeds correlated with total OSDI scores ( $\mathrm{p} \leq 0.05$ for all). Ocular-

158 discomfort-related and vision-related subscores were associated with slower IReST and sustained

159 silent reading ( $\mathrm{p} \leq 0.05$ for both), but not for the MNRead passage. As compared to those with 
$161 \mathrm{CI}=-31$ to $-7, \mathrm{p}=0.003)$ and sustained silent reading $(26 \%$ slower, $95 \% \mathrm{CI}=-38$ to $-13 \%$, $162 \mathrm{p}<0.001)$.

Additional multivariable models were run to determine the association between ocular

164 surface measures and reading speed (Table 3). Worse-eye TBUT was not significantly associated

165 with reading speed for all three tests. Corneal staining was associated with changes in IReST (-2

$166 \mathrm{wpm} / 1$ unit change in staining score, $95 \% \mathrm{CI}=-3$ to $-0.3, \mathrm{p}=0.015)$ and sustained silent reading

167 speeds $(-2 \% / 1$ unit change in staining score, $95 \% \mathrm{CI}=-4$ to $-0.6, \mathrm{p}=0.009)$, but not with

168 maximum reading speed calculated from the MNRead test $(\mathrm{p}=0.93)$. African American race and

169 lower MMSE score were significantly associated with reduced reading speed for at least one

170 reading test.

171 Multivariate GEE models (using the exchangeable correlation structure) assessing the

172 time required to read individual word/post-word interval durations demonstrated that higher

173 OSDI $(+1.1 \mathrm{~ms} / 1$ point increase in OSDI; $95 \% \mathrm{CI}=0.6$ to $1.5 ; \mathrm{p}<0.001)$ and corneal staining

174 scores $(+3.0 \mathrm{~ms} / 1$ point increase in corneal staining; $95 \% \mathrm{CI}=0.1$ to $5.8 ; \mathrm{p}=0.045)$, but not

175 TBUT or Schirmer's ( $\mathrm{p}>0.05$ for both), were associated with longer word/post-word interval

176 complex durations. Greater word/post-word interval durations were also associated with

177 increased word size, word frequency, and word location (end of line versus any other location)

$178 \quad(\mathrm{p}<0.05$ for all $)$.

Interactions between dry eye severity and text features on word/post-word interval

180 durations were also analyzed in separate multivariate GEE models for each dry eye metric. Each

181 interaction model included the metric of dry eye severity, word feature of interest, interaction

182 term (dry eye metric x word feature), and all relevant non-visual metrics. Significant interactions 
183 were noted between greater OSDI score and both word length $(\mathrm{p}=0.002)$ and word frequency

$184(p=0.02)$, but not with any other dry eye measures or features $(p>0.05$ for all).(Table 4$)$

186 DISCUSSION

In this clinic-based patient population, dry eye was associated with reduced reading 188 speeds using a variety of reading tests. This decrement correlated directly with the severity of 189 symptoms as measured with OSDI. Individuals with severe dry eye symptoms (OSDI score $>33$ )

190 had substantial reductions in sustained silent reading ( $26 \%$ decrease in wpm). These findings

191 suggest that dry eye symptoms impair reading performance, and likely interfere with daily 192 activities for which reading is critical.

193 Previous studies have demonstrated the functional impact of dry eye on various everyday 194 tasks, such as reading.[5-10,31-32] We previously demonstrated self-reported difficulty with 195 reading in an elderly population-based cohort.[12] In that study, dry eye did not significantly 196 affect reading speed, although dry eye subjects reported reading difficulty and avoidance of 197 newspaper reading. This discrepancy can be attributed to the fact that subjects from our prior 198 study were derived from a population-based sample who are likely to have less severe disease, 199 compared to the patients in the current study who were cared for at a tertiary dry eye center. 200 Additionally, in our previous study, reading speed was only measured using short out-loud text 201 passages. Finally, limited objective measures were available to categorize the severity of the dry 202 eye in our prior work.

Only two other studies to our knowledge have evaluated reading speed in dry eye. One

204 study used the Wilkins Rate of Reading test, which consists of simple words without context that 205 are read aloud and takes less than 2 minutes to complete.[33, 34] Dry eye subjects exhibited 
slower reading speeds $(134.9 \pm 4.95 \mathrm{wpm})$ than controls $(158.3 \pm 8.40 \mathrm{wpm}, \mathrm{p}=0.046)$, but were

207 not undergoing treatment at the time of evaluation which may have resulted in a larger difference

208 in reading speed than we observed. Another recent small-scale case-control study reported

209 slower reading rates in dry eye patients as well, but its association with subjective or objective

210 measures of dry eye disease was not studied.[35] Our study improves on the methodology of

211 prior studies by using reading tests that more closely mimic reading scenarios which patients

212 encounter in their day-to-day lives.

An interesting finding in our study was that the impact of dry eye on reading speed

214 differed based on the type of reading test employed. Of the two out-loud reading tests, the

215 magnitude of the associations found between dry eye measures and IReST reading speed was

216 greater as compared to MNRead maximum reading speed. One possible reason for this

217 difference is that dry eye exerts its impact on reading speed through visual disturbances that were

218 not identified in the current study (our groups had similar distance/reading acuity and contrast

219 sensitivities). MNRead reading speeds are modeled as the maximum reading speed observed for

220 the sentences presented at different text sizes, and perhaps larger text size can overcome the

221 visual disturbances associated with dry eye. We found a greater impact of dry eye on sustained

222 silent reading speed. In multivariable models, dry eye was associated with 14\% slower sustained

223 silent reading ( $20 \mathrm{wpm}$ decrement at the mean reading speed, $\mathrm{p}=0.03$ ), while the reduction with

224 IReST testing was $15 \mathrm{wpm}(\mathrm{p}=0.04)$. Our findings therefore support the validity and utility of

225 sustained silent reading speed as an important measure to evaluate patients with dry eye

226 disease.[22] Finally, our interaction analysis showed that dry eye patients do not appear to have

227 particular difficulty with word-specific features, in contrast to what has been demonstrated in the

228 glaucoma population.[24] These results suggest that dry eye disease likely affects reading in a 
more diffuse manner, as opposed to a distinct process which manifests with particular text features (i.e. peripheral visual constriction in glaucoma patients leading to particular difficulty during line transitions). For example, decreased ocular optical qualities due to dry eye disease (i.e. those captured by dynamic aberrometry) may represent the mechanism of decreased reading speed.[36] Therefore, visual rehabilitation may be more difficult to specifically tailor to the dry eye population as compared to other ocular conditions.

The limitations of our study include the fact that a great majority of the participants were on topical therapy (artificial tears, anti-inflammatories, or intraocular pressure-lowering drops), which was not held prior to enrollment. It is possible that the overall reading disability measured here is understated, given that dry eye patients were getting appropriate therapy that was not held prior to testing. The participants represented a convenience sample; therefore, perhaps less symptomatic dry eye patients were less likely to participate, biasing our findings in a positive direction. Also, patients with best-corrected visual acuity (BCVA) better than 20/40 were included, but may have had other pathology influencing reading speed. However there was no statistical difference in the BCVA between the two groups, and the associations were observed to exist independent of BCVA. Additionally, we included glaucoma suspects as controls and not individuals without any signs of dry eye. We considered the possibility that using this control group could bias our findings towards the null hypothesis if reading speed was affected by eye drop therapy. However, in our sensitivity analyses we found no difference in reading speed on any of the tests between controls using eye drops to those who did not. In addition, controls who attend essentially the same clinic as cases are more likely to be similar on unmeasured factors. Recruitment of entirely normal controls (i.e. spouses or friends accompanying patients to clinic) would likely exclude individuals who are less likely to venture outside the home due to poorer 
252 general health, mood, or cognitive ability, thus producing a "supranormal" group of controls.

253 Also our data collection did not include blink frequency, which could affect reading time and dry

254 eye measurements. Our findings pertain to a specific set of office-based environmental testing

255 conditions, and the effect of dry eye on reading may differ under other conditions such as higher

256 or lower humidity or air drafts or different lighting conditions. Future studies may consider

257 using dynamic aberrometry of the tear film in the future, which could be utilized as a

258 standardized surrogate marker and potentially facilitate multicenter clinical trials.[37]

259 In summary, our findings provide direct evidence for the impact of dry eye on reading

260 performance. Our results show that reading speed could be utilized as a tool to directly measure

261 functional impairment from dry eye. 
Table 1. Characteristics of subjects with physician-diagnosed dry eye versus controls participating in reading evaluations.

\begin{tabular}{|c|c|c|c|}
\hline & $\begin{array}{c}\text { Control* } \\
(\mathbf{n}=50)\end{array}$ & $\begin{array}{c}\text { Dry Eye } \\
(n=41)\end{array}$ & $\begin{array}{c}p \\
\text { value }\end{array}$ \\
\hline \multicolumn{4}{|l|}{ Demographics } \\
\hline Mean age, years (SD) & $67.4(8.5)$ & $65.7(10.3)$ & 0.42 \\
\hline African-American, n (\%) & $9(18.0)$ & $3(7.3)$ & 0.13 \\
\hline Female, $\mathrm{n}(\%)$ & $29(58.0)$ & $37(90.2)$ & 0.001 \\
\hline Education, years (SD) & $15.6(2.1)$ & $15.1(1.9)$ & 0.25 \\
\hline Employed, n (\%) & $24(48.0)$ & $16(39.0)$ & 0.39 \\
\hline \multicolumn{4}{|l|}{ Vision } \\
\hline Better-eye acuity, logMAR, mean (SD) & $-0.01(0.11)$ & $0.02(0.10)$ & 0.18 \\
\hline Binocular $\log \mathrm{CS}$, mean (SD) & $1.93(0.12)$ & $1.88(0.16)$ & 0.11 \\
\hline Cataract/PCO, either eye, n (\%) & $4(8.0)$ & $6(14.6)$ & 0.31 \\
\hline \multicolumn{4}{|l|}{ Health } \\
\hline MMSE Score, mean (SD) & $27.6(1.5)$ & $26.8(2.4)$ & 0.06 \\
\hline Depressive symptoms, $\mathrm{n}(\%)$ & $3(6.0)$ & $6(14.6)$ & 0.17 \\
\hline \multicolumn{4}{|l|}{ Dry Eye Measures (SD) } \\
\hline Mean OSDI Total score & $4.7(3.8)$ & $39.5(21.1)$ & $<0.001$ \\
\hline Mean OSDI Discomfort subscore & $2.8(2.8)$ & $22.2(12.2)$ & $<0.001$ \\
\hline Mean OSDI Vision subscore & $1.8(2.1)$ & $17.3(1.9)$ & $<0.001$ \\
\hline Mean TBUT in worse eye & $3.3(3.0)$ & $1.9(2.0)$ & 0.01 \\
\hline Mean corneal staining score in worse eye & $5.2(3.8)$ & $7.4(3.6)$ & 0.007 \\
\hline Mean Schirmer's test in worse eye & $10.2(9.2)$ & $8.6(9.2)$ & 0.41 \\
\hline
\end{tabular}

*Control patients included were glaucoma suspects without any history or symptoms of dry eye disease

CS: Contrast sensitivity; logMAR: Logarithm of the minimum angle of resolution; MMSE: Mini Mental State Exam; OSDI: Ocular Surface Disease Index; PCO: Posterior capsular opacity (in pseudophakic subjects); SD: Standard deviation; TBUT: Tear film break up time 
Table 2. Comparison of reading parameters in subjects with physician-diagnosed dry eye versus controls: Unadjusted values.

\begin{tabular}{|lccc|}
\hline & $\begin{array}{c}\text { Control* } \\
(\mathbf{n = 5 0})\end{array}$ & $\begin{array}{c}\text { Dry Eye } \\
(\mathbf{n = 4 1})\end{array}$ & $\begin{array}{c}\mathbf{p} \\
\text { value }\end{array}$ \\
\hline $\begin{array}{l}\text { Out-loud reading, MNRead acuity card (SD) } \\
\text { Mean maximum reading speed, wpm }\end{array}$ & $186(21)$ & $180(25)$ & 0.22 \\
$\quad$ Mean critical print size & $0.14(0.16)$ & $0.21(0.21)$ & 0.08 \\
$\quad$ Mean reading acuity, logMAR & $-0.05(0.11)$ & $-0.01(0.15)$ & 0.09 \\
$\begin{array}{l}\text { Out loud reading, IReST passage } \\
\text { Mean reading speed (SD) wpm }\end{array}$ & & & $\mathbf{1 4 8}(\mathbf{2 7})$ \\
$\begin{array}{l}\text { Sustained silent reading passage (SD) } \\
\text { Median reading speed, wpm }\end{array}$ & $\mathbf{1 6 3 ( 2 2 )}$ & & $\mathbf{0 . 0 0 6}$ \\
\end{tabular}

*Control patients included were glaucoma suspects without any history or symptoms of dry eye disease

IReST: International Reading Speed Text; MNRead card: The Minnesota low vision reading test; SD: Standard deviation; wpm: words per minute 
Table 3. Associations between MNRead, IReST, and sustained silent reading speeds with dry eye status, vision, demographic, and health variables in subjects with physician diagnosed dry eye versus controls: Multivariable analyses

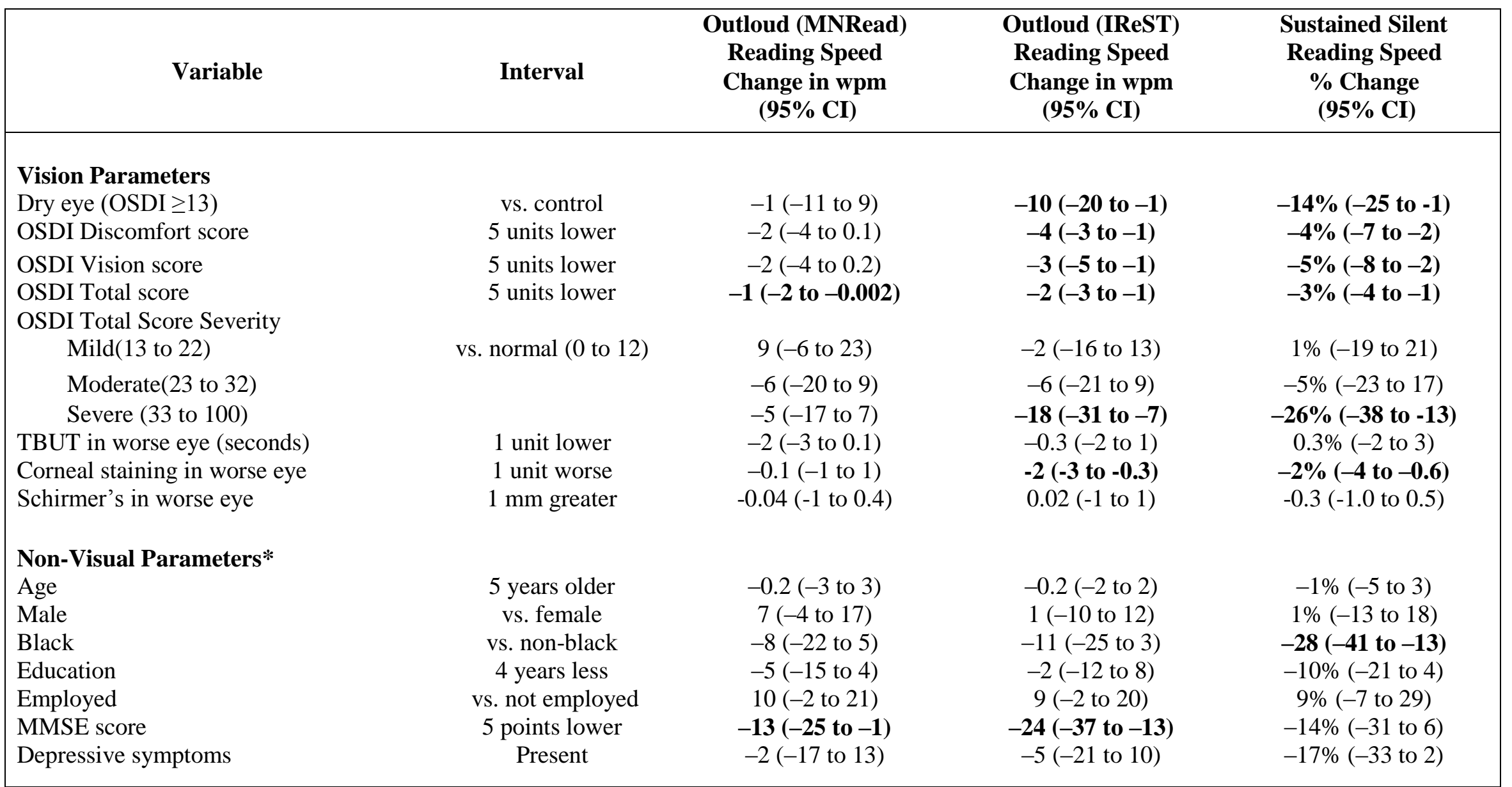

Bolded values represent statistical significance $(\mathbf{p}<0.05)$

*The values for non-visual parameters taken from a single model including dry eye covariate and all nonvisual variables are shown. All other visual parameter values were derived from a separate multivariable model including the non-visual variables shown.

IReST: International Reading Speed Text; OSDI: Ocular Surface Disease Index; SD: Standard deviation; TBUT: Tear film break-up time; wpm: Words per minute 
Table 4: Significant Interactions between Dry Eye Severity and Word Features on Word/Post-Word Interval Complex Duration, Multivariable

2 Analysis*

\begin{tabular}{|c|c|c|c|c|c|}
\hline$\underline{\text { Variable }}$ & $\underline{\text { Interval }}$ & $\begin{array}{c}\underline{\text { OSDI }} \\
\text { Word/Post-Word } \\
\text { Interval Complex (ms) } \\
\beta(95 \% \mathrm{CI})\end{array}$ & $\begin{array}{c}\text { Tear Break Up Time } \\
\text { Word/Post-Word } \\
\text { Interval Complex (ms) } \beta \\
(95 \% \mathrm{CI})\end{array}$ & $\begin{array}{l}\text { Corneal Staining } \\
\text { Word/Post-Word } \\
\text { Interval Complex } \\
(\mathrm{ms}) \beta(95 \% \mathrm{CI})\end{array}$ & $\begin{array}{l}\frac{\text { Schirmer's Test }}{\text { Word/Post-Word }} \\
\text { Interval Complex } \\
(\mathrm{ms}) \beta(95 \% \mathrm{CI})\end{array}$ \\
\hline \multicolumn{6}{|l|}{ Dry Eye \& Word Size } \\
\hline Dry eye metric* & 1 unit increase & $0.2(-0.5$ to 0.9$)$ & $2(-4$ to 7$)$ & $0.1(-4$ to 4$)$ & $0.3(-1.2$ to 2$)$ \\
\hline Word Size & 1 letter longer & 23 (19 to 28$)$ & 29 (24 to 34$)$ & $24(18$ to 29$)$ & $28(23$ to 33$)$ \\
\hline Dry eye metric $\bullet$ Word Size ${ }^{\dagger}$ & & $0.2(0.1$ to 0.3$)$ & $-0.6(-1$ to 0.3$)$ & $0.7(-0.1$ to 1.4$)$ & $-0.1(-0.3$ to 0.2$)$ \\
\hline \multicolumn{6}{|l|}{ Dry Eye \& Word Frequency } \\
\hline Dry eye metric* & 1 unit increase & $2(1$ to 3$)$ & $-2(-7$ to 4$)$ & $5(0.2$ to 10$)$ & $-0.2(-2$ to 2$)$ \\
\hline Word Frequency & 10 fold less common & 44 (41 to 48$)$ & $46(43$ to 49$)$ & $45(40$ to 49$)$ & $46(43$ to 50$)$ \\
\hline Dry eye metric $\cdot$ Word Frequency ${ }^{\dagger}$ & & $0.1(0.02$ to 0.2$)$ & $0.1(-0.6$ to 0.8$)$ & $0.3(-0.3$ to 0.9$)$ & $0.02(-0.2$ to 0.2$)$ \\
\hline \multicolumn{6}{|l|}{ Dry Eye \& Last Word of Line } \\
\hline Dry eye metric* & 1 unit increase & $1(0.5$ to 1.5$)$ & $-1(-4$ to 3$)$ & $3(-0.4$ to 5$)$ & $-0.3(-1$ to 1$)$ \\
\hline Last Word of Line & vs. not last word & $30(7$ to 53$)$ & $44(18$ to 71$)$ & $21(-9$ to 51$)$ & $44(17$ to 70$)$ \\
\hline Dry eye metric $\bullet$ Last Word of Line ${ }^{\dagger}$ & & $0.4(-0.4$ to 1$)$ & $-2(-7$ to 4$)$ & $3(-1$ to 8$)$ & $-0.5(-2$ to 1$)$ \\
\hline
\end{tabular}

Bolded values represent outcomes with $\mathbf{p}<\mathbf{0 . 0 5}$. Positive values indicate slower reading (longer word/post-word interval complex reading times) for words that

4 were longer, less frequently used, or found at the end of a line of text for the respective dry eye metric. Negative values represent faster reading (shorter

5 word/post-word interval complex reading time).

$6 *$ Four dry eye metrics used: OSDI (unit= 1 point), Tear Film Breakup Time (unit=1 second), Corneal Staining (unit= 1 point), and Schirmer's Test (unit= 1

7 millimeter).

$8 \quad$ The impact of each interaction derived from a separate model including the dry eye metric, the word feature of interest, the interaction term (dry eye metric $\mathrm{x}$

9 word feature), and all relevant non-visual metrics (age, gender, race, education, mini-mental state exam, word size, word frequency).

10 ₹ Represented by negative log of word frequency per million words used in common English language

11 CI- Confidence interval; mm- millimeter 
12 Funding: EY018595, EY022976, and Dr. Akpek has been supported in part by an unrestricted

13 grant provided by Jerome L. Greene Sjögren's Center, Johns Hopkins University, Baltimore,

14 Maryland.

15

16 Competing Interests:

17 Esen Akpek: Allergan (Irvine, CA); Advisory boards supported by Biogen Idec (Cambridge,

18 MA), Seattle Genetics (Bothell, WA), Novartis (New York, NY) \& Shire (Lexington, MA);

19 Unpaid member of Sjogren's Syndrome Foundation.

20 No other authors have any competing interests/disclosures.

21

22

23

24

25

26

27

28

29

30

31

32

33

34 


\section{Contributorship Statement:}

36 Priya Mathews: Acquisition/analysis/interpretation of data, drafting the manuscript, final

37 approval of version to be published, and agreeable to be accountable for all aspects of the work

38 Pradeep Ramulu: Design of the work, acquisition/analysis/interpretation of data, revising the

39 manuscript, final approval of version to be published, and agreeable to be accountable for all

40 aspects of the work

41 Bonnielin Swenor: Acquisition/analysis/interpretation of data, revising the manuscript, final

42 approval of version to be published, and agreeable to be accountable for all aspects of the work

43 Canan Utine: Design of the work, acquisition/analysis/interpretation of data, revising the

44 manuscript, final approval of version to be published, and agreeable to be accountable for all

45 aspects of the work

46 Gary Rubin: Design of the work, acquisition/analysis/interpretation, revising the manuscript,

47 final approval of version to be published, and agreeable to be accountable for all aspects of the

48 work

49 Esen Akpek: Design of the work, acquisition/analysis/interpretation of data, drafting and

50 revising the manuscript, final approval of version to be published, and agreeable to be

51 accountable for all aspects of the work 


\section{References}

53 1- Moss SE, Klein R, Klein BE. Prevalence of and risk factors for dry eye syndrome. Arch $54 \quad$ Ophthalmol 2000;118:1264-8.

55 2- Chia EM, Mitchell P, Rochtchina E, et al. Prevalence and associations of dry eye syndrome 56 in an older population: The Blue Mountains Eye Study. Clin Experiment Ophthalmol $57 \quad 2003 ; 31: 229-32$.

3- Uchino M, Nishiwaki Y, Michikawa T, et al. Prevalence and risk factors of dry eye disease in $59 \quad$ Japan: Koumi study. Ophthalmology 2011;118:2361-7.

60 4- The definition and classification of dry eye disease: Report of the Definition and

61 Classification Subcommittee of the International Dry Eye WorkShop 2007; Ocul Surf $62 \quad 2007 ; 5: 75-92$.

63 5- Buchholz P, Steeds CS, Stern LS, et al. Utility assessment to measure the impact of dry eye 64 disease. Ocul Surf 2006;4:155-161.

65 6- Pouyeh B, Viteri E, Feuer W, et al. Impact of ocular surface symptoms on quality of life in a 66 United States Veterans Affairs population. Am J Ophthalmol 2012;153:1061-66.

67 7- Vitale S, Goodman LA, Reed GF, et al. Comparison of the NEI-VFQ and OSDI 68 questionnaires in patients with Sjögren's syndrome-related dry eye. Health Qual Life $69 \quad$ Outcomes 2004;2:44.

8- Miljanović B, Dana R, Sullivan DA, et al. Impact of dry eye syndrome on vision-related quality of life. Am J Ophthalmol 2007;143:409-15.

72 9- Tong L, Waduthantri S, Wong TY, et al. Impact of symptomatic dry eye on vision-related 73 daily activities: the Singapore Malay Eye Study. Eye (Lond) 2010;24:1486-91. 
74 10- Schiffman RM, Christianson MD, Jacobsen G, et al. Reliability and validity of the Ocular $75 \quad$ Surface Disease Index. Arch Ophthalmol 2000;118:615-21.

76 11- Paulsen AJ, Cruickshanks KJ, Fischer ME, et al. Dry eye in the Beaver Dam Offspring

77 Study: Prevalence, risk factors, and health-related quality of life. Am J Ophthalmol $78 \quad 2014 ; 157: 799-806$.

79 12- van Landingham SW, West SK, Akpek EK, et al. Impact of dry eye on reading in a 80 population-based sample of the elderly: the Salisbury Eye Evaluation. Br J Ophthalmol $81 \quad 2014 ; 98: 639-44$.

82 13-Stern ME, Gao J, Schwalb TA, et al. Conjunctival T-cell subpopulations in Sjögren's and 83 non-Sjögren's patients with dry eye. Invest Ophthalmol Vis Sci 2002;43:2609.

84 14-Ferris FL, Kassoff A, Bresnick GH, et al. New visual acuity charts for clinical research. Am] 85 Ophthalmol 1982;94:91-96.

86 15- Bailey IL, Bullimore MA, Raasch TW, et al. Clinical grading and the effects of scaling. 87 Invest Ophthalmol Vis Sci 1991;32:422-32.

16- Elliott DB, Bullimore MA, Bailey IL. Improving the reliability of the Pelli-Robson contrast $89 \quad$ sensitivity test. Clin Vision Sci 1991;6:471-5.

17- Goldberg DP, Hillier VF. A scaled version of the General Health Questionnaire. Psychol

92 18-Folstein MF, Folstein SE, McHugh PR. "Mini-mental state". A practical method for grading 93 the cognitive state of patients for the clinician. J Psychiatr Res 1975;12:189-98.

94 19- Thylefors B, Chylack LT Jr, Konyama K, et al. A simplified cataract grading system. Ophthalmic Epidemiol. 2002;9:83-95. 
20- Legge GE, Ross JA, Luebker A, et al. Psychophysics of reading. VIII. The Minnesota low vision reading test. Optom Vis Sci 1989;66:843-53.

21- Hahn GA, Penka D, Gehrlich C, et al. New standardised texts for assessing reading performance in four European languages. Br J Ophthalmol 2006;90:480-4.

22- Ramulu PY, Swenor BK, Jefferys JL, et al. Description and validation of a test to evaluate sustained silent reading. Invest Ophthalmol Vis Sci 2013;54:673-80.

23- Cheung SH, Kallie CS, Legge GE, et al. Nonlinear mixed-effects modeling of MNREAD data. Invest Ophthalmol Vis Sci 2008;49:828-35.

24- Mathews PM, Rubin GS, McCloskey M, et al. Severity of vision loss interacts with wordspecific features to impact out-loud reading in glaucoma. Invest Ophthalmol Vis Sci 2015;56:1537-1545.

25- Walt J. Ocular Surface Disease Index (OSDI) Administration and Scoring Manual. Allergan, Inc: Irvine, CA;2004.

26- Miller KL, Walt JG, Mink DR, et al. Minimal clinically important difference for the ocular surface disease index. Arch Ophthalmol 2010;128:94-101.

27- Mathews PM, Ramulu PY, Friedman DS, et al. Evaluation of ocular surface disease in patients with glaucoma. Ophthalmology 2013;120:2241-8.

28- Lemp MA. Report of the National Eye Institute/Industry workshop on Clinical Trials in Dry Eyes. CLAO J 1995;21:221-32.

29- Liew M, Zhang M, Kim E, Akpek EK. Prevalence and predictors of Sjogren's syndrome in a prospective cohort of patients with aqueous-deficient dry eye. Br J Ophthalmol 2012;96:1498-503. 
118 30- Ramulu PY, West SK, Munoz B, Jampel HD, Friedman DS. Glaucoma and reading speed:

119 the Salisbury Eye Evaluation Project. Arch Ophthalmol. 2009;127:82-87.

120 31-Deschamps N, Ricaud X, Rabut G, eet al. The impact of dry eye disease on visual

121 performance while driving. Am J Ophthalmolol 2013;156:184-189.

122 32- Milijanovic B, Dana R, Sullivan DA, Schaumberg DA. Impact of dry eye syndrome on

123 vision-related quality of life. Am J Ophthalmol. 2007;143:409-415.

124 33- Ridder WH 3rd, Zhang Y, Huang JF. Evaluation of reading speed and contrast sensitivity in 125 dry eye disease. Optom Vis Sci 2013;90:37-44.

126 34- Wilkins AJ, Jeanes RJ, Pumfrey PD, Laskier M. Rate of Reading Test: Its reliability, and its 127 validity in the assessment of the effects of coloured overlays. Ophthalmic Physiol Opt $128 \quad 1996 ; 16: 491-7$.

129 35- Ousler GW, Rodriguez JD, Smith LM, Lane KJ, Heckley C, Angjeli E, Abelson MB.

130 Optimizing Reading Tests for Dry Eye Disease. Cornea. 2015;34:917-921.

131 36-Rieger G. The importance of the precorneal tear film for the quality of optical imaging. $\mathrm{Br} J$ 132 Ophthalmol. 1992;76:157-158.

133 37- Denoyer A, Rabut G, Baudouin C. Tear film aberration dynamics and vision-related quality 134 of life in patients with dry eye disease. Ophthalmology. 2012;119:1811-1818. 